\title{
Dissipation-induced instabilities in an optical cavity laser: A mechanical analog near the 1:1 resonance
}

\author{
Marcel G. Clerc and Jerrold E. Marsden \\ Control and Dynamical Systems, 107-81, Caltech, Pasadena, California 91125
}

(Received 25 March 2001; published 26 November 2001)

\begin{abstract}
The 1:1 resonance for perturbed Hamiltonian systems with small dissipative and energy injection terms has been studied. These perturbations of the 1:1 resonance exhibit dissipation induced instabilities. This mechanism allows one to show that an optical cavity with small pumping is unstable when one takes into account the dissipative effects. The Maxwell-Bloch equations are the asymptotic normal form that describe this instability when energy is injected through forcing at zero frequency. A simple mechanical system close to the 1:1 resonance has been displayed, which is a mechanical analog of the laser.
\end{abstract}

DOI: 10.1103/PhysRevE.64.067603

\section{INTRODUCTION}

Equilibria of Hamiltonian vector fields can undergo a variety of instabilities as a single bifurcation parameter is varied [1]. There are two fundamental codimension one bifurcations of Hamiltonian systems. The first is the stationary or steady state bifurcation, which is characterized by two eigenvalues merging at zero with multiplicity two [2]. The second is the 1:1 resonance, involving the collision of two pureimaginary eigenvalues (and their complex conjugates) at finite frequencies with multiplicity two [3]. These instabilities are a consequence of the fact that in the Hamiltonian case, if $\lambda$ is an eigenvalue, then [3] so is $-\lambda$. The same property is present in time reversible systems, i.e., they exhibit identical generic instabilities. Recently, the instabilities of quasireversible systems have also been characterized [4,5], in which the irreversible effects are small and can be considered as perturbative terms close to the instability. In particular, systems close to the quasireversible 1:1 resonance are described by the Maxwell-Bloch equations when the energy is injected through a forcing at zero frequency [5]. The Maxwell-Bloch equations describe the interaction of an electromagnetic field and a collection of two level atoms at an optical cavity [6].

The aim of this paper is to study the 1:1 resonance for systems in the neighborhood of a Hamiltonian one; that is, we shall consider perturbed Hamiltonian systems with dissipative and energy injection terms. Near this instability, the dissipative terms are responsible for a spectral bifurcation, i.e., the dissipation induces an instability [7]. These circumstances can occur in several mechanical systems, in nonlinear optical cavities, fluid mechanics (in the quasigeostrophic model [8]), in tubes carrying a fluid [9], in the motion of planets in celestial mechanics [10], and galactic dynamics [11], to mention a few.

Because of the extensive variety of application areas, the contributions of this paper are of wide appeal to many important subjects. To illustrate our general methods, we shall consider, as a specific example simple mechanical and laser systems. In these systems, the dissipation induced instability enables us to show that an optical cavity with small pumping is unstable when one takes into account the dissipative effects. The Maxwell-Bloch equations are the asymptotic normal form that describes this instability in the presence of a conserved quantity. We shall display a simple mechanical
PACS number(s): 42.60.Da, 47.52. $+\mathrm{j}, 05.45 . A c$

system, which we call the mechanical laser, which, close to the 1:1 resonance, is a mechanical analog of the laser. Additional applications of the ideas in this paper will be given elsewhere.

\section{GENERAL SETUP}

For simplicity, we first consider a Hamiltonian system with two degrees of freedom $H\left(p^{i}, q_{i},\{\lambda\}\right)$, where $p^{i}$ and $q_{i}$ are canonically conjugate variables, $(i=1,2)$, and $\{\lambda\}$ is a set of parameters. Assume that there is a 1:1 resonance for an equilibrium at $\lambda=\lambda_{c}$, i.e., the spectrum at the equilibrium has a pair of pure imaginary eigenvalues of multiplicity two, say at $\pm i \Omega$. Nearby, the instability system is governed by the normal form [12]

$$
\begin{gathered}
\partial_{t} A=i \Omega A+B, \\
\partial_{t} B=i \Omega B+f\left[|A|^{2}, i\left(A B^{*}-B A^{*}\right),\left\{\lambda-\lambda_{c}\right\}\right] A \\
+i g\left[|A|^{2}, i\left(A B^{*}-B A^{*}\right),\left\{\lambda-\lambda_{c}\right\}\right] B,
\end{gathered}
$$

where $f$ and $g$ are complex polynomial functions. There is a change of variables from the given ones $\left\{p^{1}, q_{1}, p^{2}, q_{2}\right\}$ to the new ones (the complex variables $A, B$ ) of the form $\left\{p^{1}, q_{1}, p^{2}, q_{2}\right\}=A \vec{\Psi}+B \vec{\chi}+\vec{N}\left(A, B, A^{*}, B^{*}\right)$, where $\vec{\Psi}$ is the eigenvector of the linearized system corresponding to $i \Omega$ and $\vec{\chi}$ is a generalized eigenvector in the Jordan sense, and $\vec{N}\left(A, B, A^{*}, B^{*}\right)$ is a nonlinear vector. This change of variables is not canonical in general. When one considers rotated variables $\left(A=e^{i \Omega t} A^{\prime}, B=e^{i \Omega t} \partial_{t} A^{\prime}\right)$ and the dominant terms, the normal form reads (omitting the primes)

$$
\partial_{t t} A=\varepsilon A+i \delta \partial_{t} A-\alpha|A|^{2} A,
$$

where $\varepsilon$ is the bifurcation parameter, which is proportional to $\lambda-\lambda_{c}$; henceforth we assume $\varepsilon \ll 1, \delta$ is the gyroscopic term [3] or detuning term [6] and $\alpha$ is an order-one parameter. The asymptotic normal form (2) has the following Hamiltonian:

$$
H=\partial_{t} A \partial_{t} A^{*}-\varepsilon|A|^{2}+\frac{\alpha}{2}|A|^{4},
$$

with the Poisson bracket ( $F$ and $G$ are real valued) 


$$
\{F, G\}=\frac{\partial F}{\partial A} \frac{\partial G}{\partial A_{t}^{*}}-\frac{\partial G}{\partial A} \frac{\partial F}{\partial A_{t}^{*}}+i \delta \frac{\partial F}{\partial A_{t}} \frac{\partial G}{\partial A_{t}^{*}}+\text { c.c. }
$$

Thus, to cubic order, the nonlinear change of variables is canonical. The eigenvalues of the zero solution $(A=0)$ are $\pm 1 / 2 \sqrt{4 \varepsilon-2 \delta^{2} \pm 2 \delta \sqrt{\delta^{2}-4 \varepsilon}}$. When $\varepsilon-\delta^{2} / 4$ is negative, the initial Hamiltonian system has four distinct pure imaginary eigenvalues; $\varepsilon-\delta^{2} / 4$ equal to zero is the $1: 1$ resonance at $\pm i \Omega$ frequencies and when it is positive, the eigenvalues have nonzero real part. Note that the gyroscopic term is a stabilizing effect [3].

\section{ADDING DISSIPATION}

We now consider this Hamiltonian system under the influence of small dissipative terms. This leads to a new term in the asymptotic normal form as follows:

$$
\partial_{t t} A=\varepsilon A-(\mu-i \delta) \partial_{t} A-\alpha|A|^{2} A,
$$

where $\mu$ is positive. To study the effects of dissipative terms in Eq. (4), we consider its characteristic polynomial: $\lambda^{4}$ $+2 \mu \lambda^{3}+\left(\mu^{2}+\delta^{2}-2 \varepsilon\right) \lambda^{2}-\lambda 2 \varepsilon \mu+\varepsilon^{2}$. Looking for roots of the form $\lambda=a \pm i b$ and $\lambda=c \pm i d$, one recognizes that $\mu$ $=-(a+c)$ and $\varepsilon \mu=\left[c\left(a^{2}+b^{2}\right)+a\left(c^{2}+d^{2}\right)\right]$. Hence, when $\varepsilon$ is negative, $a$ and $c$ are negative, i.e., all eigenvalues are to the left of the imaginary axis. For $\varepsilon$ positive and $\varepsilon$ $-\delta^{2} / 4$ negative, the unperturbed system is marginal, but the perturbed one satisfies $a c<0$; in addition, the eigenvalues with larger frequency move to the left of the imaginary axis (stable modes) and the others to the right (unstable modes), but the eigenvalues that move furthest away from the axis are stable. Finally, when $\varepsilon>\delta^{2} / 4$, the eigenvalues have nonzero real part, and again the stable modes are the furthest from the imaginary axis.

We now consider dissipation induced instabilities [7]. The destabilizing effects through positive or negative total dissipative perturbation was known already by Thomson and Tait [3]. One can understand this phenomena as follows: when $\varepsilon$ is negative, the energy (3) has a minimum at the origin, hence when dissipation is added, the solutions near the origin move towards it. By contrast, if $\varepsilon$ is positive, the energy has a saddle point at the origin and when $\varepsilon<\delta^{2} / 4$, this is unstable with an algebraic evolution in time. We say that the system (2) exhibits a latent bifurcation [13] for $\varepsilon=0$; when one adds dissipation, this is consistent with the conclusion that the solutions near the origin move away from it, exponentially in time.

Using the preceding analysis, we infer that close to the 1:1 resonance, generically the dissipative terms induce an instability. In the case that the instability happens with null detuning $(\delta=0)$, the normal form has only a real coefficient and so the dissipative terms do not induce instability. A physical example of this last situation is the weakly dissipative baroclinic instability when the effect of earth's sphericity is ignored [14] and another is the Kelvin-Helmholz instability [15].

\section{ENERGY INJECTION}

As we shall see in detail later, the laser is a system that shows dissipation induced instability, but first, we need to discuss how the energy is injected to the system, so that the solution that becomes unstable is persistent when the dissipative terms are added. There are two natural ways to inject energy to the modes, namely, through forcing at finite frequency or at zero. The latter situation is common in physical systems. In a Hamiltonian system this is only possible if there is a conserved quantity, that is, a zero eigenvalue whose mode is nonlinearly coupled with the other ones. For instance, when there is a cyclic variable, the respective momentum $Z$ is conserved. A Hamiltonian system that has a 1:1 resonance in the presence of conserved quantity, leads us to consider an extra equation of the form $\partial_{t} Z=0$ and an additional term $-Z A$ in Eq. (4). The system presents different behaviors depending on the value of $\epsilon-Z$. When one includes dissipative terms and forcing, the asymptotic normal form reads

$$
\begin{gathered}
\partial_{t t} A=(\varepsilon-Z) A-(\mu-i \delta) \partial_{t} A-\alpha|A|^{2} A, \\
\partial_{t} Z=\nu Z+\eta|A|^{2} .
\end{gathered}
$$

The term $\eta|A|^{2}$ permits a nontrivial coupling between the variables. When the unperturbed Hamiltonian system has more modes without resonances between them, the perturbed system is governed by the above equations, since the intensities of the other modes decreases in time. Through a nonlinear change of variables, the previous equations are equivalent to the Maxwell-Bloch equations [5]. Using a multiscaling method, the dispersive instability with small dissipation is also described by the previous equations [16].

\section{EXAMPLE: THE LASER}

To illustrate how dissipation induced instabilities enter, we consider the semiclassical description of the laser. This is based on the self-consistent interaction of the electromagnetic field with an active medium within an optical cavity. The electric field is described classically (by the Maxwell equations) and the matter as ensemble of atoms possessing two quantized energy levels; phenomenological terms are added to complete the description. Thus, the system is described by $[6,17]$

$$
\begin{gathered}
\frac{\partial^{2} E}{\partial t^{2}}=\frac{\partial^{2} E}{\partial x^{2}}-\frac{\partial^{2} P}{\partial t^{2}}-\kappa \frac{\partial E}{\partial t}, \\
\frac{\partial^{2} P}{\partial t^{2}}=-\gamma_{\perp} \frac{\partial P}{\partial t}-\left[\gamma_{\perp}^{2}+(1+\delta)^{2}\right] P-\mu^{2} N E, \\
\frac{\partial N}{\partial t}=-\gamma_{\|}\left(N-N_{0}\right)+E\left(\frac{\partial P}{\partial t}+\gamma_{\perp} P\right),
\end{gathered}
$$

with periodic boundary condition at the cavity length $[L, E(0, t)=E(L, t)]$. Here $E, P$, and $N$ are dimensionless quantities, that correspond to linearly polarized electric field, 

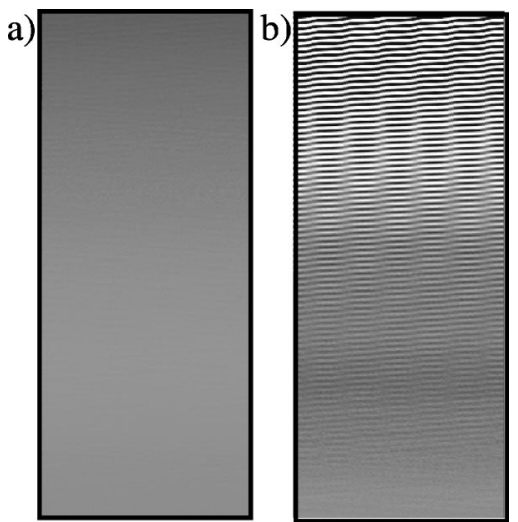

FIG. 1. Spatiotemporal diagram of the electric field of the semiclassical model [Eq. (6)] with $L=120, \quad \delta=0.94, \quad g=0.6, \quad D_{o}$ $=0.0338$. (a) Without dissipation $\kappa=\gamma_{\perp}=\gamma_{\|}=0$, (b) with dissipation $\kappa=0.03, \gamma_{\perp}=0.01, \gamma_{\|}=0.03$, and $N_{o}=0.0338$.

the dipole polarization field and the population inversion. $\gamma_{\|}, \quad \gamma_{\perp}$ are the decay rate associated to spontaneous emission and interaction between the atoms, $\kappa$ is a damping related to the mirror losses, $\delta$ is the detuning, $\mu$ is a coupling constant which characterizes the atoms and $N_{0}$ the pump parameter. In the time reversible limit of the above equations, i.e., $\gamma_{\perp}=\gamma_{\|}=\kappa=0$, the system has, after an appropriate scaling, the Hamiltonian density

$$
\mathcal{H}=\frac{1}{2}\left[D-\left(\frac{\mu}{\Omega}\right)^{2} P\right]^{2}+\frac{\left(\partial_{x} A\right)^{2}}{2}+\left(\frac{\mu}{\Omega}\right)^{2} N
$$

with the Poisson bracket

$$
\begin{aligned}
\{F, K\}= & \int d x\left\{\frac{\partial F}{\partial A} \frac{\partial K}{\partial D}-\frac{\partial F}{\partial D} \frac{\partial K}{\partial A}\right. \\
& \left.+\left(\frac{\mu}{\Omega}\right)^{2} \vec{m} \cdot\left(\vec{\nabla}_{m} F \times \vec{\nabla}_{m} K\right)\right\},
\end{aligned}
$$

where $\vec{m}=\left(N, P, \partial_{t} P\right)$ and $E=\partial_{t} A$ and $\Omega=1+\delta$. The Hamiltonian is just the sum of the electromagnetic energy and the atomic excitation energy.

Changing the cavity length in the time reversible limit of Eq. (6), leads to a 1:1 resonance for the nonlasing solution $\left(E=P=0, N=D_{0}\right)$, which gives rise to an electromagnetic wave with $\pm \Omega$ frequencies. Using the slowly varying envelope (WKB) approximation leads to the Maxwell-Bloch equations [6].

Figure 1 shows the space-time diagram of the electric field of a numerical simulation of the semiclassical model, plus noise with small intensity, close to the 1:1 resonance without dissipative terms [see Fig. 1(a)] and with dissipative ones [see Fig. 1(b)]. The numerical simulations start with the same initial condition, namely, the no-lasing solution with excited atoms $\left(D_{0}>0\right)$.

It is clear from these pictures that the inclusion of dissipation induces the laser to respond. One can physically understand what happens, since without dissipation the atoms excited decay through the stimulated emission, i.e., the non-

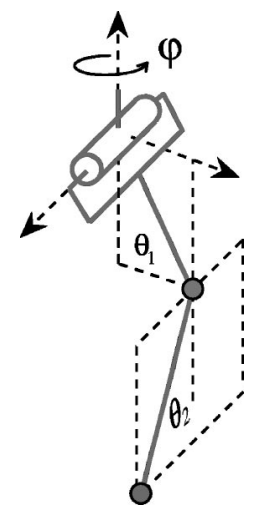

FIG. 2. Schematic representation of the mechanical laser.

lasing solution becomes unstable very slowly (nonlinear mechanism). Instead, when one takes into account the dissipative terms, the excited atoms decay, for instance through stimulated emission and collisions, i.e., exponentially in time. Note that, one must pump to $N_{0}=D_{0}$ so that the nonlasing solution persists. Using the Energy-Casimir method [2] it is easy to show that the nonlasing solution has a latent bifurcation for $D_{0}=0$, this shows that with nonzero detuning and slight pumping, the dissipation induces a laser response.

\section{MECHANICAL LASER}

To illustrate the 1:1 resonance in a simple Hamiltonian system, we consider a mechanical system, that we call the mechanical laser, which consists of two coupled spherical pendula in a gravitational field, with a support, which can rotate around a vertical axis. The lower pendulum is constrained to move in a plane that is orthogonal to the plane of the upper pendulum (see Fig. 2).

The system rotates with angular velocity $\dot{\varphi}$ with respect to the vertical. The quantities $m_{1}, m_{2}, l_{1}$, and $l_{2}$ are the mass and length of the upper and lower pendula, respectively, and $I$ is the dimensionless moment of inertia of the support. The system will dissipate energy because of friction at the contacts and the motion of the pendulum masses in a fluid (for example, the air) via Stokes's law. Energy is injected through a constant torque at the upper pendulum pivot point. The governing equations for the angles $\theta_{1}(t)$ and $\theta_{2}(t)$ and the vertical angular velocity $\dot{\varphi}$ read [13]

$$
\begin{aligned}
\ddot{\theta}_{1}= & -\sigma^{2} \sin \theta_{1} \sin \theta_{2} \ddot{\theta}_{2}-\sigma^{2} \sin \theta_{1} \cos \theta_{2} \dot{\theta}_{2}^{2} \\
& -2 \sigma^{2} \cos \theta_{1} \cos \theta_{2} \dot{\varphi}_{2}+\sin \theta_{1} \cos \theta_{1} \dot{\varphi}^{2} \\
& -\sigma^{2} \cos \theta_{1} \sin \theta_{2} \ddot{\varphi}-\frac{g}{l} \sin \theta_{1}-\nu_{1} \dot{\theta}_{1},
\end{aligned}
$$

$$
\begin{aligned}
\ddot{\theta}_{2}= & -\sin \theta_{1} \sin \theta_{2} \ddot{\theta}_{1}-\cos \theta_{1} \sin \theta_{2} \dot{\theta}_{1}^{2}+2 \cos \theta_{1} \cos \theta_{2} \dot{\varphi} \dot{\theta}_{1} \\
& +\sin \theta_{2} \cos \theta_{2} \dot{\varphi}^{2}+\sin \theta_{1} \cos \theta_{2} \ddot{\varphi}-\frac{g}{l} \sin \theta_{2}-\nu_{2} \dot{\theta}_{2},
\end{aligned}
$$




$$
\begin{aligned}
& \frac{d}{d t}\left\{\begin{array}{c}
\left(\sin ^{2} \theta_{1}+\sigma^{2} \sin ^{2} \theta_{2}\right) \dot{\varphi}+\sigma^{2} \cos \theta_{1} \sin \theta_{2} \dot{\theta}_{1} \\
-\sigma^{2} \sin \theta_{1} \cos \theta_{2} \dot{\theta}_{2}+I \dot{\varphi}
\end{array}\right\} \\
& \quad=-\nu_{\varphi}(\dot{\varphi}-\Omega)-\mu_{1} \sin ^{2} \theta_{1} \dot{\varphi}-\mu_{2}\left(\sin ^{2} \theta_{1}+\sin ^{2} \theta_{2}\right) \dot{\varphi},
\end{aligned}
$$

where $\nu_{1}, \nu_{2}, \nu_{\varphi}, \mu_{1}$, and $\mu_{2}$ are damping coefficients, $\sigma$ $=\sqrt{m_{2} /\left(m_{1}+m_{2}\right)}$ is the relative factor of the energy between the oscillators, and we have written the torque as $\nu_{\varphi} \Omega$. For the sake of simplicity, we have considered the case of pendula of equal lengths $\left(l_{1}=l_{2}=l\right)$. When one considers the Hamiltonian limit of the previous equations, the vertical solution or nonlasing solution $\theta_{1}=\theta_{2}=0, \dot{\varphi}=\Omega_{0}$ has a $1: 1$ resonance when $\Omega_{0}=\Omega_{c}=\sqrt{g\left(m_{1}+m_{2}\right) / l m_{1}}$ with frequency $\omega_{c}= \pm \sqrt{g m_{2} / l m_{1}}$. The centripetal force is more intense than the gravitational force when $\Omega_{0} \geqslant \Omega_{g} \equiv \sqrt{g / l}$. As a consequence, the Coriolis force exerted by one pendulum on the other, the nonlasing solution is marginal when $\Omega_{g} \leqslant \Omega_{0}$ $\leqslant \Omega_{c}$. In this region the system is nonlinearly unstable and the system becomes linearly unstable when $\Omega_{0}>\Omega_{c}$; this exhibits a coherence oscillation, which is the signature of the laser instability.

Near the 1:1 resonance, the coupled pendulum is governed by Eqs. (5), where [13]

$$
\begin{gathered}
\varepsilon=2 \frac{g}{l} \frac{\left(\Omega-\Omega_{c}\right)}{\Omega_{c}}, \quad \alpha=\frac{g}{4 l}\left(\frac{\sigma^{4}-2 \sigma^{3}-2 \sigma^{2}+3}{1-\sigma^{2}}\right), \\
\delta=2 \sigma\left(\Omega-\Omega_{c}\right), \quad \nu=\frac{\nu_{\varphi}}{I}, \quad \mu=\frac{1}{2 l^{2}}\left(\nu_{1}+\nu_{2}\right), \\
\eta=\frac{1}{I}\left[\mu_{1}+\left(1+\frac{1}{\sigma^{2}}\right) \mu_{2}-2 \frac{\nu_{\varphi}}{\sigma^{2} I}\left(\frac{\Omega}{\Omega_{c}}-1\right)\right]
\end{gathered}
$$

where the variables are related to the dominate order by $A$ $=\left(\sigma \theta_{1}+i \theta_{2}\right) \exp \left(i \omega_{c} t\right) / 2$ and $Z=2 \Omega_{c} \sigma^{2}\left(\Omega_{c}-\dot{\varphi}\right)$. Transforming Eq. (5) to the Maxwell-Bloch equations, one obtains the following analogy of the electric field $E=\left\{\sigma \theta_{1}\right.$ $\left.+i \theta_{2}\right\} e^{i\left(\Delta+\omega_{c}\right) t} / 2+$ c.c., $\quad$ polarization $P=\left\{\sigma \dot{\theta}_{1}+i \dot{\theta}_{2}\right.$ $\left.+a\left(\sigma \theta_{1}+i \theta_{2}\right)\right\} e^{i\left(\Delta+\omega_{c}\right) t} / 2+$ c.c. and population inversion $N$ $=2 \Omega_{c} \sigma^{2}\left(\dot{\varphi}-\Omega_{c}\right) / \alpha+\varepsilon_{0}-\left(\sigma^{2} \theta_{1}^{2}+\theta_{2}^{2}\right) / 4$, where $\Delta=\delta(\nu \alpha$ $+\eta) /(2 \nu \alpha+2 \eta-\mu \alpha), a=(\nu \alpha+\eta) / \alpha+i 2 \Delta$, and $\varepsilon_{0}=(\varepsilon$ $\left.+\Delta^{2}-\delta \Delta\right) / \alpha+(\nu \alpha+\eta)(\mu \alpha-\nu \alpha-\eta) / \alpha^{3}$. The mechanical analog of the electric field is a simple function of the pendulum displacements with respect to the vertical. In the Hamiltonian case the polarization is the slow time derivative of the electric field. The mechanical analogy of the stimulated emission mechanism (time reversal effect) is the vertical angular momentum conservation. Thus, when one increases the pendula tilt (the intensity of the electric field enlargement), the angular velocity decreases (the population inversion declines), which is a main ingredient of laser theory. If the torque or pumping is equal to the gravitational force $\left(\Omega_{0}=\Omega_{g}\right)$, the mechanical laser exhibits a latent bifurcation, and then the torque that gives rise to an angular velocity slightly higher than $\Omega_{g}$ leads to a dissipation induced instability.

Another system that exhibits dissipation induced instability is the baroclinic instability [8], for large $\beta$ effect this occurs distant of $1: 1$ resonance, this suggests the presence of latent bifurcation. Work in this direction is in progress.

\section{SUMMARY}

We have studied the 1:1 resonance for perturbed Hamiltonian systems with small dissipative and energy injection terms. Nearby, the 1:1 resonance exhibits dissipation induced instabilities. This allows us to show that a slightly pumping optical cavity is unstable when one takes into account the dissipative effects. The Maxwell-Bloch equations are the asymptotic normal form that describe this instability when energy is injected through forcing at zero frequency. We have displayed a simple mechanical system, the Mechanical laser or double spherical pendulum with a support, which, close to the 1:1 resonance, is a mechanical analog of the laser.

J.E.M. was partially supported by the National Science Foundation. M.G.C. is grateful for support from the Programa de insercion de Científicos of Fundación Andes.
[1] R. Abraham and J. E. Marsden, Foundations of Mechanics (Addison-Wesley, Reading, MA, 1978).

[2] J. E. Marsden and T. S. Ratiu, Introduction to Mechanics and Symmetry (Springer-Verlag, New York, 1994).

[3] W. Thomson and P. G. Tait, Principles of Mechanics and Dynamics (Cambridge University Press, Cambridge, England, 1962).

[4] M. Clerc, P. Coullet, and E. Tirapegui, Phys. Rev. Lett. 83, 3820 (1999).

[5] M. Clerc, P. Coullet, and E. Tirapegui, Opt. Commun. 166, 159 (1999)

[6] M. Sargent, M. O. Scully, and W. E. Lamb, Laser Physics (Addison-Wesley, Reading, MA, 1974).

[7] A. M. Bloch, P. S. Krishnaprasad, J. E. Marsden, and T. S. Ratiu, Ann. Inst. Henri Poincare 11, 37 (1994).
[8] R. D. Romea, J. Atmos. Sci. 34, 1689 (1977).

[9] A. K. Bajaj, P. R. Sethna, and T. S. Lundgren, SIAM (Soc. Ind. Appl. Math.) J. Appl. Math. 39, 213 (1980).

[10] W. S. Koon, M. W. Lo, J. E. Marsden, and S. D. Ross, Chaos 10, 427 (2000).

[11] B. P. Kondrat'ev, Astron. Rep. 44, 279 (2000).

[12] C. Elphick, E. Tirapegui, M. Brachet, P. Coullet, and G. Iooss, Physica D 29, 95 (1987).

[13] M. Clerc and J. E. Marsden (unpublished).

[14] J. Pedlosky, J. Atmos. Sci. 28, 587 (1971).

[15] M. Weissman, Philos. Trans. R. Soc. London, Ser. A 290, 639 (1979).

[16] J. D. Gibbon and M. McGuinness, Phys. Lett. 77A, 295 (1980).

[17] A. E. Seigman, Laser (University Science books, Mill Valley, CA, 1986). 